Vol.62: e19180403, 2019

http://dx.doi.org/10.1590/1678-4324-2019180403

ISSN 1678-4324 Online Edition

Article - Biological and Applied Sciences

\title{
Evaluation of Reference Genes for Quantitative PCR in Four Tissues from Rabbits with Hypercholesterolaemia
}

Zhen Zhang ${ }^{1}$

https://orcid.org/0000-0002-3305-7666

Bin Wen ${ }^{1}$

https://orcid.org/0000-0003-4798-111X

Yuan $\mathrm{Xu} \mathbf{u}^{1}$

https://orcid.org/0000-0002-2837-2952

En-ze Jiang 1

https://orcid.org/0000-0003-3705-1189

Jia-yu Liu ${ }^{1}$

https://orcid.org/0000-0003-1403-7448

Ke-li Zhu ${ }^{1}$

https://orcid.org/0000-0002-5963-875X

Fang-yong Ning ${ }^{1}$

https://orcid.org/0000-0003-4211-5917

Zhi-Heng Du ${ }^{1}$

https://orcid.org/0000-0002-8006-0446

Xiu-Juan Bai ${ }^{*}$

https://orcid.org/0000-0002-4233-707X

${ }^{1}$ Northeast Agricultural University, College of Animal Science and Technology, Harbin, Heilongjiang, China.

Received: 2018.07.31; Accepted: 2019.07.08.

*Correspondence: Xiu-Juan Bai bxiujuan630306@163.com; (X.J.B.) 


\section{HIGHLIGHTS}

- HLW male rabbits appear suitable for producing a model of hypercholesterolaemia.

- Five different algorithms are used to evaluate the most stable reference genes.

- GeNorm algorithm is used for determining the optimal number of reference genes.

- The most stable reference genes for the model showed tissue specificity.

Abstract: Rabbit with hypercholesterolaemia is an important model for studying cholesterol metabolism disease. This study aimed to evaluate the expression stability of nine reference genes for quantitative PCR ( $\mathrm{PPCR}$ ) analysis in adrenal gland, liver, spleen, and kidney tissue from rabbits with hypercholesterolaemia. In total, 30 male Harbin Large White (HLW) rabbits were fed a normal feed $(n=15)$ or a high cholesterol feed $(n=15)$ for 8 weeks to induce hypercholesterolaemia. Nine reference genes were verified by qPCR using cDNA extracted from rabbit tissue samples. For qPCR analysis, reference genes were evaluated using the RefFinder and GeNorm algorithms. Overall, seven rabbits with hypercholesterolaemia were identified based on body weight and total cholesterol measurements. Combining the results of the RefFinder and GeNorm algorithms, the most stable reference genes were hypoxanthine phosphoribosyltransferase 1 (Hprt1) and eukaryotic translation elongation factor 1 alpha 1 (Eef1a1) in the adrenal gland, $\beta$-2-microglobulin (B2m) and glyceraldehyde-3-phosphate dehydrogenase (Gapdh) in the liver, tyrosine 3-monooxygenase/tryptophan 5-monooxygenase activation protein zeta (Ywhaz) and Gapdh in the spleen, and peptidylprolyl isomerase (Ppia), $\beta$-actin (Actb), succinate dehydrogenase complex subunit A flavoprotein (Sdha), and B2m in the kidney. Taken together, our results confirmed that Hprt1 and Eef1a1, B2m and Gapdh, Ywhaz and Gapdh, and Ppia, Actb, Sdha, and B2m were the best reference genes for qPCR analyses in adrenal gland, liver, spleen, and kidney tissue, respectively, of rabbits with hypercholesterolaemia.

Keywords: Evaluation; reference genes; qPCR; rabbits; hypercholesterolaemia.

\section{INTRODUCTION}

Rabbit models have significantly contributed to human disease research for many years. As a common human chronic disease, obesity can lead to hypercholesterolaemia, which in itself is associated with other serious diseases [1]. To study diseases associated with hypercholesterolaemia in humans, it is essential to produce an effective animal model of disease [2]. Because of their physiological characteristics, a rabbit model of hypercholesterolaemia can be produced easily, rapidly, and inexpensively through the feeding of a high cholesterol diet for a relatively short period of time [3].

A high cholesterol feed is important for the production of a rabbit hypercholesterolaemia model. In New Zealand White rabbits, one of the most common breeds worldwide, induction of hypercholesterolaemia is affected by cholesterol levels in the feed [4]. A cholesterol level below $1 \%$ is one of the most important factors for inducing hypercholesterolaemia, and allows the development of an excellent model to study severe human diseases such as atherosclerosis, myocardial infarction, and stroke [5]. In addition to cholesterol levels in feed, breed, age, sex, and feeding time can also affect the induction of hypercholesterolaemia in 
rabbits [3]. However, with careful consideration of the above factors, the rabbit hypercholesterolaemia model can be very valuable for use in human disease research.

Quantitative PCR (qPCR) is the most widely used experimental method for detecting gene expression, and is currently one of the most accurate, sensitive, and specific methods available [6]. However, many factors can influence the reliability of qPCR analysis, including purity and quality of RNA, reverse-transcriptase efficiency, and PCR efficiency [7]. To account for any error introduced by these factors, many normalization methods use reference genes to allow comparison between samples [8]. Reference genes are used as an internal control and are considered to be expressed at a constant level under different experimental conditions. However, recent studies have shown that many widely used reference genes such as Gapdh and Actb are not stably expressed under different experimental conditions $[9,10]$, indicating no single reference gene is universal. Therefore, it is essential to evaluate the most suitable reference genes for the specific experimental conditions to obtain more accurate results.

In some rabbit models, a select group of reference genes are commonly used for qPCR analysis [11]. For these analyses, the most stable reference genes have been evaluated in specific tissues using different algorithms. Several early reports from mouse models used five different algorithms (GeNorm, BestKeeper, NormFinder, the $\Delta \mathrm{Ct}$ approach, and RefFinder) to accurately evaluate the most stable reference genes [12,13]. More recently, the most stable reference genes in heart tissue from rabbits with left ventricular diastolic dysfunction were validated using two different algorithms (GeNorm and NormFinder) [11]. However, no previous studies have examined the most stable reference genes in specific tissues from rabbits with hypercholesterolaemia. Therefore, the aim of current study was to accurately evaluate the most stable reference genes from amongst nine different functional reference genes, in accordance with two previous studies related to rabbits [11,14], for qPCR analysis in four tissues (adrenal gland, liver, spleen, and kidney) from rabbits with hypercholesterolaemia using five different algorithms (GeNorm, BestKeeper, NormFinder, the $\Delta \mathrm{Ct}$ approach, and RefFinder).

\section{MATERIALS AND METHODS}

\section{Ethics statement}

All animal work was carried out in accordance with the guidelines for the Care and Use of Experimental Animals established by the Ministry of Science and Technology of the People's Republic of China (Approval number: 2006 - 398) and was also approved by the Laboratory Animal Management Committee at Northeast Agricultural University, Harbin, China.

\section{Production of rabbits with Hypercholesterolaemia}

Thirty male Harbin Large White (HLW) rabbits (body weight: $2.0 \pm 0.25 \mathrm{~kg}$ ) were used in the current study. These rabbits were fed a normal diet $(0 \%$ cholesterol $+0 \%$ fat $+0 \%$ yolk powder $+100 \%$ normal feed) and housed in individual cages for 1 week prior to experimentation to allow for acclimatization. The environment was maintained at a temperature of $24 \pm 2^{\circ} \mathrm{C}$ and all animals had free access to water. After 1 week, the 30 rabbits were randomly divided into two groups: control group and hypercholesterolaemia group. The control group rabbits $(n=15)$ were fed a normal diet (as above) for 8 weeks, while the hypercholesterolaemia group rabbits $(n=15)$ were fed a high cholesterol diet $(1 \%$ cholesterol $+10 \%$ fat $+10 \%$ yolk powder $+79 \%$ normal feed) for the same time period to induce hypercholesterolaemia.

Hypercholesterolaemia was confirmed through body weight and total cholesterol measurements taken at the end of the experimental period (8 weeks). The weight of each rabbit was measured prior to drawing blood, while total cholesterol was detected from blood serum samples extracted from the venous blood of rabbits that had fasted for $12 \mathrm{~h}$. 


\section{Tissue collection, total RNA extraction, and cDNA synthesis}

After blood samples were collected, all rabbits (control, $n=15$; hypercholesterolaemia, $n=7$ ) were euthanized via cervical dislocation to collect four tissues (adrenal gland, liver, spleen, and kidney). All tissues were frozen and stored in liquid nitrogen for extraction of total RNA.

Total RNA was extracted from all tissue samples using a Roche (Mannheim, Germany) High Pure RNA Tissue Kit with DNase I treatment according to the manufacturer's instructions. The concentration and quality of extracted total RNA from each sample was determined by spectrophotometer (BioPhotometer D30; Eppendorf, Hamburg, Germany), with a 260/280 absorbance ratio of 1.8-2.1 indicating that the RNA was suitable for subsequent cDNA synthesis.

cDNA synthesis was performed using a Roche Transcriptor First Strand cDNA Synthesis Kit according to the manufacturer's instructions. cDNA was synthesized using 1 $\mu \mathrm{g}$ of total RNA extracted from each of the tissue samples from the control $(n=15)$ and hypercholesterolaemia $(n=7)$ rabbits. The cDNA was diluted 1:5 for qPCR analyses.

\section{Selection of reference genes and primers and determination of primer efficiencies}

Nine reference genes, suitable primers, and reported primer efficiencies were identified from previous literature relating to rabbits $[11,14]$. To validate the nine selected reference genes and their primers and to confirm primer efficiencies, cDNA generated from the four types of tissue samples (adrenal gland, liver, spleen, and kidney) from the control $(n=15)$ and hypercholesterolaemia $(n=7)$ rabbits was examined by qPCR. Serial dilutions of the synthesized cDNA mixtures were used to calculate the efficiency of each set of primers targeting the nine reference genes. qPCR assays were performed using an ABI 7500 Real Time PCR System (Life Technologies, Carlsbad, CA, USA). Reactions were carried out in a total volume of $10 \mu \mathrm{l}$ containing $0.8 \mu \mathrm{l}(1.6 \mathrm{ng})$ of cDNA, $0.5 \mu \mathrm{l}(5 \mathrm{pmol})$ of forward PCR primer, $0.5 \mu \mathrm{l}(5 \mathrm{pmol})$ of reverse PCR primer, $5 \mu \mathrm{l}$ of FastStart Universal SYBR Green Master (ROX) (Roche), and $3.2 \mu \mathrm{l}$ of UltraPure nuclease-free water. The reaction conditions were as follows: $95^{\circ} \mathrm{C}$ for $10 \mathrm{~min}$, followed by 40 cycles of $95^{\circ} \mathrm{C}$ for $15 \mathrm{~s}$ and $60^{\circ} \mathrm{C}$ for $1 \mathrm{~min}$. All reactions were performed in 96-well plates in triplicate technical replicates. Primer efficiencies were determined following examination of cycle threshold values (Cq values) using 7500 software (Life Technologies).

Further qPCR assays were conducted to determine $\mathrm{Cq}$ values for each of the nine reference genes in all four tissue types (adrenal gland, liver, spleen, and kidney) from both the control $(n=15)$ and hypercholesterolaemia $(n=7)$ rabbits. Average Cq values were calculated using Excel software to evaluate the nine reference genes in each of the tissues. In addition, primer efficiencies within a range of $86.513-106.675 \%$ were calculated using 7500 software (Life Technologies) (Table 1).

Table 1. Validation of the efficiencies of primer sets targeting nine reference genes in rabbits with hypercholesterolaemia. 


\begin{tabular}{|c|c|c|c|c|c|}
\hline $\begin{array}{l}\text { Gene } \\
\text { Symbol }\end{array}$ & $\begin{array}{c}\text { Accession } \\
\text { number }\end{array}$ & Primer sequence $\left(5^{\prime}-3^{\prime}\right)$ & $\begin{array}{l}\mathrm{Tm} \\
\left({ }^{\circ} \mathrm{C}\right)\end{array}$ & $\begin{array}{l}\text { Amplicon } \\
\text { Length } \\
\text { (bp) }\end{array}$ & $\begin{array}{l}\text { Primer } \\
\text { efficiency } \\
(\%)^{1}\end{array}$ \\
\hline Actb & NM_001101683 & $\begin{array}{l}\text { Forward: ATCAGCAAGCAGGAGTATGAC } \\
\text { Reverse: GCCAATCTCGTCTCGTTTCT } \\
\text { [14] }\end{array}$ & 87.3 & 135 & 93.893 \\
\hline$B 2 m$ & XM_002717921 & $\begin{array}{l}\text { Forward:AACGTGGAACAGTCAGACC } \\
\text { Reverse:AGTAATCTCGATCCCATTTC } \\
{[14]}\end{array}$ & 82.5 & 157 & 96.768 \\
\hline Eef1a1 & NM_001082339 & $\begin{array}{l}\text { Forward:TTGGCTACAACCCTGACACA } \\
\text { Reverse: GGTGACTTTCCATCCCTTGA } \\
\text { [14] }\end{array}$ & 83.4 & 110 & 100.711 \\
\hline Gapdh & NM_001082253 & $\begin{array}{l}\text { Forward: ATGGTGAAGGTCGGAGTGAA } \\
\text { Reverse: GGGTGGAATCATACTGGAACA } \\
{[14]}\end{array}$ & 84.6 & 151 & 106.675 \\
\hline Hprt1 & NM_001105671 & $\begin{array}{l}\text { Forward:CCTTGGTCAAGCAGTATAATC } \\
\text { Reverse: GGGCATATCCTACAACAAAC } \\
\text { [11] }\end{array}$ & 77.8 & 135 & 97.864 \\
\hline Ppia & NM_001082057 & $\begin{array}{l}\text { Forward:TCTCACCCACCTGACCATTC } \\
\text { Reverse: GCAGACACGGAACCAAAGAC } \\
\text { [14] }\end{array}$ & 86.3 & 107 & 86.513 \\
\hline Rpl5 & NM_001195679 & $\begin{array}{l}\text { Forward: GATTGCGTATGCCCGTATAG } \\
\text { Reverse: CTCCAGTCACCTCCACTTG } \\
\text { [11] }\end{array}$ & 80.2 & 194 & 99.108 \\
\hline Sdha & XM_002723194 & $\begin{array}{l}\text { Forward:GGACCAGGACGCCATCCACTAC } \\
\text { Reverse:TCCACCGAACGCACGCTGATAG } \\
\text { [11] }\end{array}$ & 79.3 & 124 & 96.928 \\
\hline Ywhaz & XM_002721227 & $\begin{array}{l}\text { Forward:CCAGGGAGATGAAGGAGATG } \\
\text { Reverse: TCGCACAAAGGGATGTATGT } \\
\text { [14] }\end{array}$ & 81.7 & 106 & 100.244 \\
\hline
\end{tabular}

Actb: $\beta$-actin, cytoskeleton; B2m: $\beta$-2-microglobulin, immune system; Eef1a1: eukaryotic translation elongation factor 1 alpha 1, protein synthesis; Gapdh: glyceraldehyde-3-phosphate dehydrogenase, carbohydrate metabolism; Hprt1: hypoxanthine phosphoribosyltransferase 1, purine metabolism; Ppia: peptidylprolyl isomerase, cyclosporin binding protein/inhibitor of serine-threonine phosphatase; Rpl5: ribosomal protein L5, ribosomal protein; Sdha: succinate dehydrogenase complex, subunit A, flavoprotein, antioxidant enzyme and phosphorylation pathway; Ywhaz: tyrosine 3-monooxygenase/tryptophan 5-monooxygenase activation protein, zeta, signal transduction.

${ }^{1}$ The primer efficiencies were calculated using 7500 software (Life Technologies).

\section{Evaluation of the nine reference genes}

To accurately evaluate the nine reference genes within the same tissue type based on the Cq values, the RefFinder algorithm was used to obtain the ranking order of the genes, while the GeNorm algorithm was used to calculate the optimal number of reference genes for each tissue type. 
The ranking order of the nine reference genes was calculated using five different algorithms (GeNorm, BestKeeper, NormFinder, the $\Delta \mathrm{Ct}$ approach, and RefFinder) using an online RefFinder tool (http://150.216.56.64/referencegene.php). The GeNorm algorithm determines the ranking order of reference genes $(n=$ the number of reference genes, $n=3$ or $n>3$ ) based on gene stability measurements ( $M$ values) that are calculated from intragroup pairwise variation (within each sample) with gene expression ratios from transformation of $\mathrm{Cq}$ values. It indicates that the ranking order of $\mathrm{M}$ values corresponds to the stability order of reference genes. Overall, the lowest $M$ value corresponds to the most stable reference gene [15]. The BestKeeper algorithm determines the ranking order of reference genes $(n=10$ or $n<10)$ from the standard deviation $(S D, S D<1)$ of the descriptive statistics of crossing points values ( $C_{p}$ values, which are equated to $\mathrm{Cq}$ values [7]) that are used for the pairwise correlation analyses. It indicates that the ranking order of SD values corresponds to the stability order of reference genes. Similarly, the lowest SD value corresponds to the most stable reference gene [16]. The NormFinder algorithm identifies the ranking order of reference genes $(n=8$ or $n>8)$ based on the stability values from the combination of intragroup variation (within each sample) and intergroup variation (within each reference gene) based on $\mathrm{Cq}$ values. It indicates that the ranking order of the stability values corresponds to the stability order of reference genes. Again, the lowest stability value corresponds to the most stable reference gene [17]. The $\Delta \mathrm{Ct}$ approach determines the ranking order of reference genes $(n=3$ or $n>3)$ from the mean standard deviation (mean $\mathrm{SD}$ ), which is calculated from the intragroup $\Delta \mathrm{Ct}$ values ( $\Delta \mathrm{Cq}$ values) of "pairs of reference genes". It indicates that the ranking order of mean SD values corresponds to the stability order of reference genes, with the lowest mean SD value corresponding to the most stable reference gene [18].

Using the data generated from these four algorithms, RefFinder determines the ranking order of reference genes from the comprehensive values that are calculated from the geometric mean (GM) values determined by the four different algorithms. It indicates that the ranking order of GM values corresponds to the stability order of reference genes. As such, the lowest GM value corresponds to the most stable reference gene [19].

In addition, the optimal number of reference genes for use in each tissue type was determined based on $\mathrm{M}$ values determined using the GeNorm algorithm. This algorithm determines the optimal number of reference genes in accordance with a value of $V_{n / n+1}<$ 0.150 ( $n=$ the optimal number of reference genes, with 0.150 representing a threshold value defined by the GeNorm algorithm). The value of $\mathrm{V}_{n / n+1}$ is calculated from $\mathrm{NF}_{n}$ and $\mathrm{NF}_{n+1}(\mathrm{NF}$, normalization factors), whereby $\mathrm{NF}_{n}$ and $\mathrm{NF}_{n+1}$ represent the $\mathrm{GM}$ of $\mathrm{M}_{n}$ and $\mathrm{M}_{n+1}$, respectively.

$\mathrm{NF}_{n}=$

while $\mathrm{NF}_{n+1}=$

$$
\sqrt[n]{\mathrm{M}_{1} \times \mathrm{M}_{2} \times \mathrm{M}_{3} \times \cdots \cdots \mathrm{M}_{n}}
$$

$$
\sqrt[n+1]{\mathrm{M}_{1} \times \mathrm{M}_{2} \times \mathrm{M}_{3} \times \cdots \cdots \mathrm{M}_{n+1}},
$$

where $M_{n}$ and $M_{n+1}=M$ values are obtained from the GeNorm algorithm, and $n=a$ serial number of $\mathrm{M}_{n}[15]$.

\section{Statistical analysis}

A Student's $t$-test was used to analyse the body weight and total cholesterol data from both groups of rabbits at the end of the 8-week feeding period. A value of $p<0.01$ was considered statistically significant.

\section{RESULTS}




\section{Induction of Hypercholesterolaemia in rabbits}

At the end of the 8-week feeding period, the body weights and total blood cholesterol levels of all rabbits (control group, $n=15$; hypercholesterolaemia group, $n=15$ ) were assessed. The results showed that seven of the 15 rabbits from the hypercholesterolaemia group had significantly higher body weights $(3.49 \pm 0.26 \mathrm{~kg})$ and total cholesterol levels $(3.02 \pm 0.87 \mathrm{mmol} / \mathrm{l})$, on average, than the 15 control group rabbits (average body weight, $2.89 \pm 0.13 \mathrm{~kg}$; average total cholesterol, $1.63 \pm 0.24 \mathrm{mmol} / \mathrm{l})(p<0.01$, Figure 1). These results indicated that hypercholesterolaemia was successfully induced in rabbits by feeding a high cholesterol diet for 8 weeks.

A

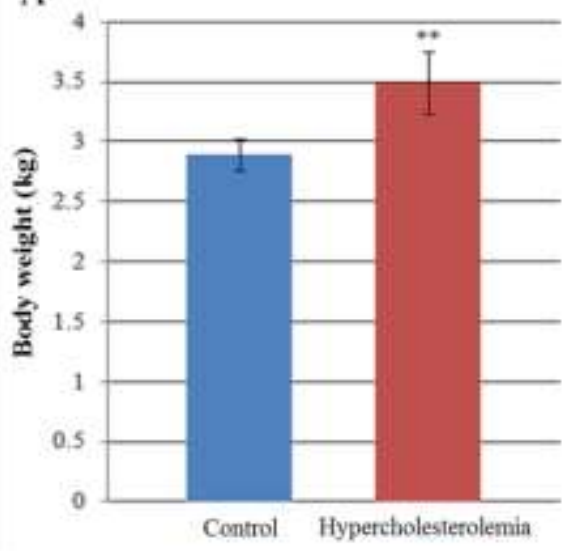

$\mathrm{B}$

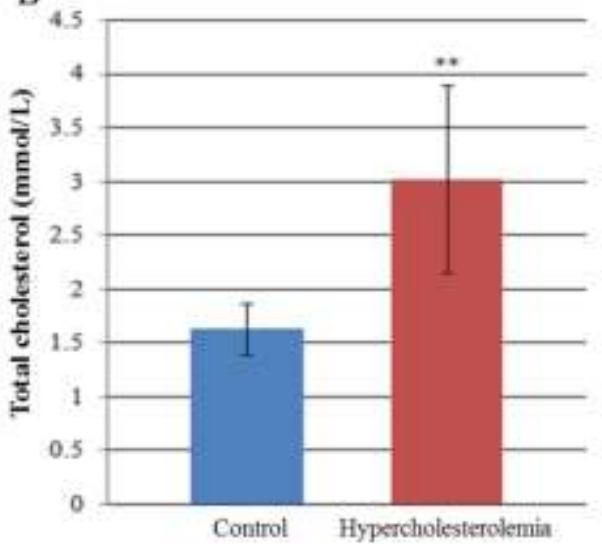

Figure 1. Confirmation of hypercholesterolaemia in rabbits through measurement of body weight and total cholesterol. (A) The average body weight $(2.89 \pm 0.13 \mathrm{~kg})$ of the control group was significantly lower than that of the hypercholesterolaemia group $(3.49 \pm 0.26 \mathrm{~kg}) .{ }^{* *}$, significantly different by Student's $t$-test, $p<0.01$. Control, $\mathrm{n}=15$; hypercholesterolaemia, $\mathrm{n}=7$. (B) The average total cholesterol level $(1.63 \pm 0.24 \mathrm{mmol} / \mathrm{l})$ of the control group was significantly lower than that of the hypercholesterolaemia group $(3.02 \pm 0.87 \mathrm{mmol} / \mathrm{l}) .{ }^{* *}$, significantly different by Student's $t$-test, $p<$ 0.01 . Control, $n=15$; hypercholesterolaemia, $n=7$.

\section{Identification of the most stable reference genes in each tissue type}

The top four reference genes for each of the tissue types identified using the five algorithms are listed in Table 2. More detailed ranking orders of the nine reference genes in the four tissues can be found in supplementary data Figure S1.

\section{Adrenal gland}

Based on the data shown in Table 2, the top four reference genes in adrenal gland tissue as determined by RefFinder were: Hprt1, Eef1a1, Rp/5, and Ppia. Three of the top four genes determined by the GeNorm and $\Delta \mathrm{Ct}$ analyses overlapped with those obtained using RefFinder (Eef1a1, Rp/5, and Ppia), while only two of the genes identified using BestKeeper and NormFinder matched those identified using RefFinder (Hprt1 and Eef1a1).

\section{Liver}

The top four reference genes in liver tissue as determined by RefFinder were Gapdh, B2m, Ywhaz, and Eef1a1. BestKeeper and $\Delta$ Ct each identified three genes overlapping with those identified by RefFinder (B2m, Ywhaz, and Eef1a1 and Gapdh, Eef1a1, and Ywhaz, respectively), with GeNorm and NormFinder identifying two overlapping genes each ( $Y$ whaz and Eef1a1 and Gapdh and Eef1a1, respectively). 
RefFinder analysis of the data shown in Table 2 showed that the top four reference genes in spleen tissue were Ywhaz, Gapdh, Ppia, and Sdha. The results of the GeNorm and $\Delta \mathrm{Ct}$ analyses were identical to those of RefFinder, while BestKeeper and NormFinder each identified two overlapping reference genes (Gapdh and Ppia and Ywhaz and Sdha, respectively).

\section{Kidney}

Based on the data shown in Table 2, the top four reference genes as determined by RefFinder were Ppia, Actb, Sdha, and B2m. GeNorm, NormFinder, and $\Delta$ Ct analyses each identified three reference genes overlapping with those identified using RefFinder (Ppia, $A c t b$, and Sdha), while BestKeeper identified two overlapping genes (B2m and Sdha).

Table 2. Top four most stable reference genes in each tissue type by analysis method

\begin{tabular}{|c|c|c|c|c|c|}
\hline & RefFinder & GeNorm & BestKeeper & NormFinder & $\begin{array}{c}\text { The } \Delta \mathrm{Ct} \\
\text { approach }\end{array}$ \\
\hline $\begin{array}{l}\text { Adrena } \\
\text { I gland }\end{array}$ & $\begin{array}{c}\text { Hprt1 }>\text { Eef1a1 } \\
> \\
\text { Rpl5 }>\text { Ppia }\end{array}$ & $\begin{array}{c}\text { Eef1a1 }=\text { Rpl5 } \\
> \\
\text { Ppia > Ywhaz }\end{array}$ & $\begin{array}{c}\text { Ppia }>\text { Ywhaz } \\
> \\
\text { Rpl5 > } \\
\text { Eef1a1 }\end{array}$ & $\begin{array}{l}\text { Hprt1 > B2m > } \\
\text { Actb }>\text { Eef1a1 }\end{array}$ & $\begin{array}{c}\text { Hprt1 > Eef1a1 } \\
>B 2 m>\text { Actb }\end{array}$ \\
\hline Liver & $\begin{array}{l}\text { Gapdh }>\text { B2m }> \\
Y w h a z>\text { Eef1a1 }\end{array}$ & $\begin{array}{c}\text { Ywhaz }=\text { B2m } \\
>\text { Ppia }> \\
\text { Eef1a1 }\end{array}$ & $\begin{array}{c}\text { B2m > Ywhaz } \\
>\text { Ppia }> \\
\text { Eef1a1 }\end{array}$ & $\begin{array}{c}\text { Gapdh > Eef1a } \\
>\text { Sdha > Ppia }\end{array}$ & $\begin{array}{l}\text { Gapdh }>\text { Eef1a1 } \\
>\text { Ppia }>\text { Ywhaz }\end{array}$ \\
\hline Spleen & $\begin{array}{c}\text { Ywhaz > Gapdh } \\
>\text { Ppia> Sdha }\end{array}$ & $\begin{array}{c}\text { Gapdh=Ppia } \\
>Y w h a z> \\
\text { Sdha }\end{array}$ & $\begin{array}{c}\text { B2m>Gapdh } \\
>\text { Ppia }> \\
\text { Eef1a1 }\end{array}$ & $\begin{array}{c}\text { Ywhaz }>\text { Sdha } \\
>\text { Eef1a1 }> \\
\text { Actb }\end{array}$ & $\begin{array}{c}\text { Ywhaz> Sdha } \\
>\text { Gapdh> Ppia }\end{array}$ \\
\hline Kidney & $\begin{array}{l}\text { Ppia }>\text { Actb }> \\
\text { Sdha }>\text { B2m }\end{array}$ & $\begin{array}{l}\text { Ppia = Actb > } \\
\text { Gapdh > Sdha }\end{array}$ & $\begin{array}{c}\text { Ywhaz > B2m } \\
>\text { Sdha }> \\
\text { Gapdh }\end{array}$ & $\begin{array}{l}\text { Ppia }>\text { Actb> } \\
\text { Sdha > Hprt1 }\end{array}$ & $\begin{array}{l}\text { Ppia }>\text { Sdha }> \\
\text { Actb }>\text { Gapdh }\end{array}$ \\
\hline
\end{tabular}

\section{Optimal number of reference genes in each tissue type}

In accordance with the cut-off value of 0.150 suggested by the GeNorm algorithm, the pairwise variation $V_{2 / 3}$ values in the adrenal gland, liver, and spleen were $V_{2 / 3}=0.139, V_{2 / 3}=$ 0.110 , and $V_{2 / 3}=0.124$, respectively, all of which were $<0.150$, confirming that the optimal number of reference genes in these tissues was two (Figure 2). In the kidney, the pairwise variation values were $\mathrm{V}_{2 / 3}=0.184, \mathrm{~V}_{3 / 4}=0.182$, and $\mathrm{V}_{4 / 5}=0.125(<0.150)$, indicating that the optimal number of reference genes in the kidney was four (Figure 2 ). 


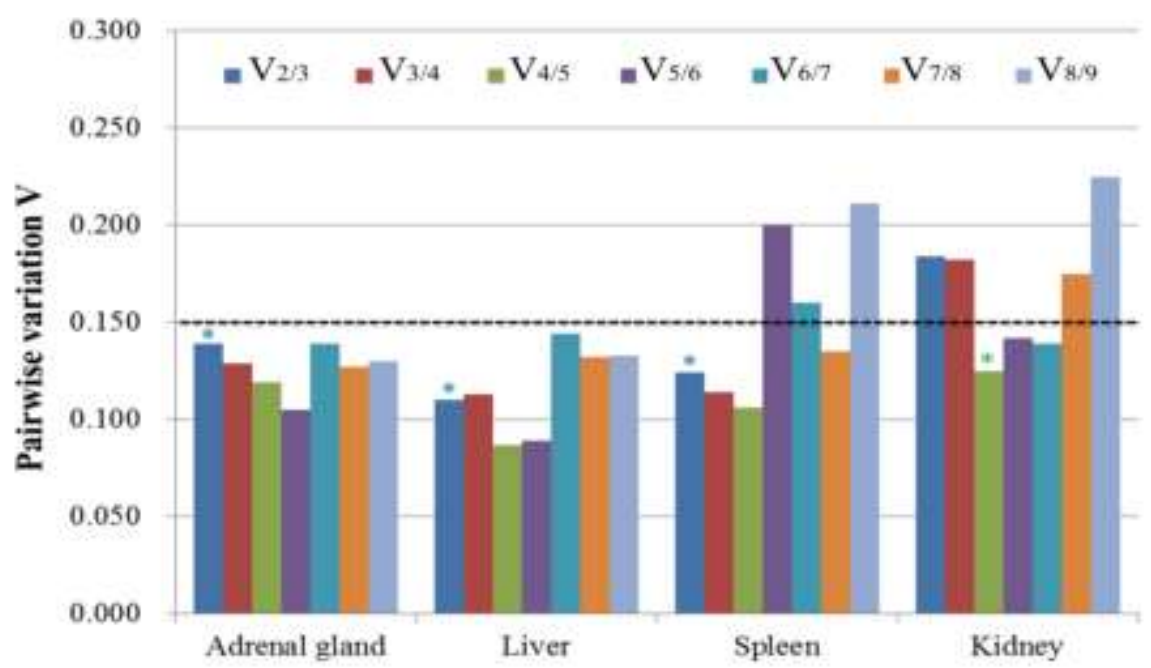

Figure 2. Determination of the optimal number of reference genes in each of the four tissues using the GeNorm algorithm. The optimal number of reference genes was determined from the pairwise variation $\left(\mathrm{V}_{n / n+1}<0.150, n=\right.$ the optimal number of reference genes). The dotted line indicates the threshold value $(0.150)$ defined by the GeNorm algorithm. Asterisks represent values of $V_{n / n+1}<$ 0.150 , which are indicative of the optimal number of reference genes $(n)$ in a given tissue. The optimal number of reference genes in the adrenal gland, liver, spleen, and kidney was determined by a value of $\mathrm{V}_{2 / 3}\left(\mathrm{~V}_{2 / 3}=0.139<0.150, n=2\right),\left(\mathrm{V}_{2 / 3}=0.110<0.150, n=2\right),\left(\mathrm{V}_{2 / 3}=0.124<0.150, n=2\right)$, and $\left(\mathrm{V}_{4 / 5}\right.$ $=0.125<0.150, n=4)$, respectively.

\section{Evaluation of the nine reference genes in the four tissues using RefFinder and GeNorm}

The combined results of the ranking order and optimal number of reference genes analyses were used to evaluate the most stable, and therefore suitable, reference genes in each of the four tissues examined in this study.

For the three tissues for which two reference genes were determined to be optimal, the most suitable reference genes were Hprt1 (GM value $=2.236$ ) and Eef1a1 (GM value $=$ 2.378 ) in the adrenal gland, Gapdh (GM value $=2.236)$ and $B 2 m(G M$ value $=2.449)$ in the liver, and Ywhaz (GM value $=1.968)$ and Gapdh (GM value $=2.060)$ in the spleen (Figure 3). In the kidney, for which four reference genes were determined to be optimal, the most suitable reference genes were Ppia $(\mathrm{GM}$ value $=1.495)$, Actb (GM value $=2.449)$, Sdha $(\mathrm{GM}$ value $=2.913)$, and $B 2 m(\mathrm{GM}$ value $=3.976)($ Figure 3$)$.

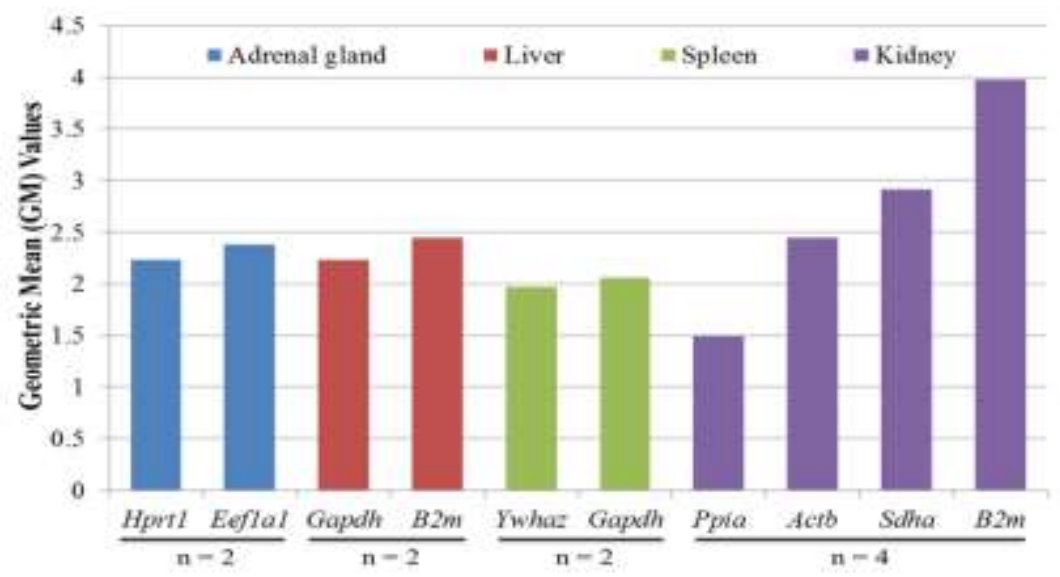

Figure 3. Evaluation of the most stable reference genes in each of the four tissues using RefFinder and GeNorm. The ranking order of reference genes based on geometric mean (GM) values (as determined by RefFinder) and the calculation of the optimal number of the reference genes based on 
the pairwise variation (determined by GeNorm) were: Hprt1 > Eef1a1 $(n=2)$ in the adrenal gland, Gapdh $>$ B2m $(n=2)$ in the liver, Ywhaz $>$ Gapdh $(n=2)$ in the spleen, and Ppia $>$ Actb $>$ Sdha $>$ B2m $(n=4)$ in the kidney.

\section{DISCUSSION}

The development of a rabbit hypercholesterolaemia model is very valuable for the study of human diseases associated with hypercholesterolaemia [2]. In most cases, hypercholesterolaemia is related to a high cholesterol diet in humans [20]. Because rabbits are sensitive to cholesterol, high cholesterol feeds can also induce hypercholesterolaemia in these model animals [3]. In the current study, HLW male rabbits, a common native breed in China, were fed a diet containing $1 \%$ cholesterol for 2 months, leading to a gradual increase in total cholesterol levels (maximum level of $3.02 \pm 0.87 \mathrm{mmol} / \mathrm{l}$ ) that corresponded to hypercholesterolaemia in the affected rabbits. In contrast, New Zealand White male rabbits fed a diet containing $1 \%$ cholesterol for more than 1 month showed rapid increases in total cholesterol (25.9 mmol/l), which was detrimental to the study of hypercholesterolaemia [4]. Therefore, the HLW male rabbit hypercholesterolaemia model used in the current study could prove valuable for future research on chronic diseases in humans.

In recent years, several research groups have developed algorithms, including GeNorm [15], BestKeeper [16], NormFinder [17], the $\Delta$ Ct approach [18], and RefFinder [19], to identify the most stable reference gene(s) in a set of samples. Using these algorithms, the stability of various reference genes under specific experimental conditions has been evaluated in many studies of animals and plants [21-23]. Although all five algorithms are suitable, RefFinder and GeNorm are considered the best algorithms for evaluation of the most stable reference gene(s) because RefFinder yields more accurate results and GeNorm determines the optimal number of reference genes needed for normalization of qPCR data. The other three algorithms are not comprehensive enough or will only identify one stable reference gene, as has been covered by previous studies and reviews related to the evaluation of stable reference gene(s) in various organisms [6,22-25].

Using RefFinder is essential for determining the accurate ranking order of reference genes [13,26]. The RefFinder algorithm uses data generated by four other algorithms, including GeNorm, BestKeeper, NormFinder, and the $\Delta \mathrm{Ct}$ approach, and can be used to determine the ranking order of reference genes [19]. In the current study, the ranking order of the nine reference genes as determined by the RefFinder algorithm significantly differed from the orders determined by the other four algorithms (GeNorm, BestKeeper, NormFinder, and the $\Delta \mathrm{Ct}$ approach) in the same tissue types (Figure S1A-D). For example, in the adrenal gland, the ranking order of the nine reference genes was Hprt1 > Eef1a1 > Rpl5 > Ppia > B2m > Ywhaz > Actb > Gapdh > Sdha by RefFinder, but this order did not match the outputs of the other four algorithms (Figure S1A). The significant differences between RefFinder and the other four algorithms likely result from the fact that RefFinder calculates a comprehensive value based on the data generated by the four other algorithms [19], while each of the other algorithms calculate individual values based on their own set of data. Undoubtedly, RefFinder more accurately determines the ranking order of reference genes.

The GeNorm algorithm is indispensable for determining the optimal number of reference genes for use in a given tissue [15]. The algorithm can accurately determine the optimal number of reference genes in accordance with a value of $\mathrm{V}_{n / n+1}<0.150(n=$ the optimal number of reference genes). In the current study, the GeNorm algorithm determined that the optimal number of reference genes was two in the adrenal gland, liver, and spleen, and four in the in the kidney (Figure 2 and 3 ). In the kidney, only the $V_{4 / 5}$ value was less than the threshold value of 0.150 , indicating that four reference genes were optimal. In contrast, a $\mathrm{V}_{2 / 3}$ value $<0.150$ was obtained in all other tissues, indicating that two reference genes were sufficient. Therefore, the GeNorm algorithm could determine the optimal number of reference genes in each of the tissue types examined in the current study.

The stability of reference genes is affected by several factors, including tissue type and specific experimental conditions [6,27]. As shown in many previous studies, these factors may have different impacts on the expression of reference genes, meaning that some 
reference genes are more stable than others in a particular tissue under specific conditions $[7,8,27,28]$. In a study related to hypercholesterolaemic rabbits, Nachar et al. found that, in left ventricular tissue, Hprt1, Gapdh, Sdha, and Rpl5 were the best reference genes for qPCR normalization [11]. However, the results of our analysis of reference genes in adrenal gland, liver, spleen, and kidney tissue using a similar model were not in agreement with these previous results. In addition, a study of rabbit bone marrow mesenchymal stem cells by Ma et al. demonstrated that Ywhaz, Ppia, and Gapdh were the best reference genes to study chondrogenic differentiation, while $R p / 13 a$ was more appropriate for analysis of osteogenic differentiation, again showing that reference gene stability varies depending on tissue type and experimental conditions [14].

The results of our study showed both differences and similarities to findings produced by studies conducted in other rodent models. In the adrenal gland, Hprt1 and Eef1a1 were the most stable reference genes in the current study; however, Hprt1 ranked last in an analysis of reference genes in a mouse model by Rok Kosir et al [29]. It was noteworthy though that Gapdh and $B 2 m$ were identified as the most stable reference genes in the liver in the present study, as previous studies have also identified Gapdh [30] and B2m [31] as being the most stable reference genes in the liver in mouse models of obesity, with similar results also obtained by Matouskova et al [31]. Herein, Ywhaz and Gapdh were identified as the most stable reference genes in the spleen, which does not agree with results of previous studies in mouse models [32,33]. Ppia, Actb, Sdha, and B2m showed the greatest stability in the kidney tissue of hypercholesterolaemic rabbits, which supports a previous study of appropriate reference genes in kidney tissue that also found Actb to be one of the best reference genes [34].

In addition, the current study showed that Actb, one of the most commonly used reference genes in qPCR-based analyses, was only identified as a stable reference gene in the kidney, echoing a previous report using a mouse model of obesity [30]. Overall, like many previous studies $[6,7,22]$, we suggest using no fewer than two reference genes for normalization, which results in more reliable gene expression analysis data.

\section{CONCLUSION}

In conclusion, HLW male rabbits appear suitable for producing a rabbit model of hypercholesterolaemia, while the combination of the RefFinder and GeNorm algorithms can be used to evaluate the most stable reference genes in different tissue types. The most stable reference genes for qPCR analysis of rabbits with hypercholesterolaemia showed tissue specificity, with Hprt1 and Eef1a1 being most suitable in the adrenal gland, Gapdh and B2m in the liver, Ywhaz and Gapdh in the spleen, and Ppia, Actb, Sdha and B2m in the kidney.

Funding: This research received no external funding.

Acknowledgments: We thank Tamsin Sheen, PhD, from Liwen Bianji, Edanz Editing China, for editing the English text of a draft of this manuscript.

Conflicts of Interest: We have no conflict of interest.

\section{REFERENCES}

1. Steinberg D. Thematic review series: the pathogenesis of atherosclerosis. An interpretive history of the cholesterol controversy: part I. J Lipid Res. 2004;45(9):1583-93.

2. Kritchevsky D. Role of cholesterol vehicle in experimental atherosclerosis. Am J Clin Nutr. 1970;23(8):1105-10.

3. Fan J, Kitajima S, Watanabe T, Xu J, Zhang J, Liu E. Rabbit models for the study of human atherosclerosis: from pathophysiological mechanisms to translational medicine. Pharmacol Ther. 2015;146:104-19. 
4. Bocan TM, Mueller SB, Mazur MJ, Uhlendorf PD, Brown EQ, Kieft KA. The relationship between the degree of dietary-induced hypercholesterolemia in the rabbit and atherosclerotic lesion formation. Atherosclerosis. 1993;102(1):9-22.

5. Li X, Liu Y, Zhang H, Ren L, Li Q, Li N. Animal models for the atherosclerosis research: a review. Protein Cell. 2011;2(3):189-201.

6. Rocha AJ, Monteiro-Júnior JE, Freire JEC, AJS S. Real Time PCR: the Use of Reference Genes and Essential Rules Required to Obtain Normalisation Data Reliable to Quantitative Gene Expression. Journal of Molecular Biology Research. 2015;5:45-55.

7. Bustin SA, Benes V, Garson JA, Hellemans J, Huggett J, Kubista M. The MIQE guidelines: minimum information for publication of quantitative real-time PCR experiments. Clin Chem. 2009;55(4):611-22.

8. Kozera B, Rapacz M. Reference genes in real-time PCR. J Appl Genet. 2013;54(4):391-406.

9. Suzuki T, Higgins PJ,Crawford DR. Control selection for RNA quantitation. Biotechniques. 2000;29(2):332-7.

10. Ruan W, Lai M. Actin, a reliable marker of internal control? Clin Chim Acta. 2007;385(1-2):1-5.

11. Nachar W, Busseuil D, Shi Y, Mihalache-Avram T, Mecteau M, Rheaume E. Optimisation of reference genes for gene-expression analysis in a rabbit model of left ventricular diastolic dysfunction. PLoS One. 2014;9(2):e89331.

12. Meyer FR, Grausgruber H, Binter C, Mair GE, Guelly C, Vogl C. Cross-platform microarray meta-analysis for the mouse jejunum selects novel reference genes with highly uniform levels of expression. PLoS One. 2013;8(5):e63125.

13. Gong ZK, Wang SJ, Huang YQ, Zhao RQ, Zhu QF, Lin WZ. Identification and validation of suitable reference genes for RT-qPCR analysis in mouse testis development. Mol Genet Genomics. 2014;289(6):1157-69.

14. Ma H, Yang Q, Li D, Liu J. Validation of suitable reference genes for quantitative polymerase chain reaction analysis in rabbit bone marrow mesenchymal stem cell differentiation. Mol Med Rep. 2015;12(2):2961-8.

15. Vandesompele J, De Preter K, Pattyn F, Poppe B, Van Roy N, De Paepe A. Accurate normalization of real-time quantitative RT-PCR data by geometric averaging of multiple internal control genes. Genome Biol. 2002;3(7):34.

16. Pfaffl MW, Tichopad A, Prgomet C, Neuvians TP. Determination of stable housekeeping genes, differentially regulated target genes and sample integrity: BestKeeper--Excel-based tool using pair-wise correlations. Biotechnol Lett. 2004;26(6):509-15.

17. Andersen CL, Jensen JL, Orntoft TF. Normalization of real-time quantitative reverse transcription-PCR data: a model-based variance estimation approach to identify genes suited for normalization, applied to bladder and colon cancer data sets. Cancer Res. 2004;64(15):5245-50.

18. Silver N, Best S, Jiang J, Thein SL. Selection of housekeeping genes for gene expression studies in human reticulocytes using real-time PCR. BMC Mol Biol. 2006;7:33.

19. Xie F, Xiao P, Chen D, Xu L, Zhang B. miRDeepFinder: a miRNA analysis tool for deep sequencing of plant small RNAs. Plant Mol Biol. 2012;80(1):75-84.

20. Steinberg D. Thematic review series: the pathogenesis of atherosclerosis. An interpretive history of the cholesterol controversy, part V: the discovery of the statins and the end of the controversy. J Lipid Res. 2006;47(7):1339-51.

21. Park SJ, Kwon SG, Hwang JH, Park DH, Kim TW.; Kim, C.W. Selection of appropriate reference genes for RT-qPCR analysis in Berkshire, Duroc, Landrace, and Yorkshire pigs. Gene. 2015;558(1):152-8.

22. Rocha AJ, Maranhao PA, Silva RO, Pohl S, Fonteles CSR. Identification of suitable reference genes for gene expression normalization in Jatropha curcas $L$ during development and under stress conditions using Real Time Quantitative PCR. Braz Arch Biol Techn. 2016;59.

23. Almeida-Oliveira F, Leandro JGB, Ausina P, Sola-Penna M, Majerowicz D. Reference genes for quantitative PCR in the adipose tissue of mice with metabolic disease. Biomed Pharmacother. 2017; 88:948-55. 
24. Gong H, Sun L, Chen B, Han Y, Pang J, Wu W. Evaluation of candidate reference genes for RT-qPCR studies in three metabolism related tissues of mice after caloric restriction. SCl REP. 2016;6.

25. Shakeel M, Rodriguez A, Bin Tahir U, Jin F. Gene expression studies of reference genes for quantitative real-time PCR: an overview in insects. Biotechnol Lett. 2018;40(2):227-36.

26. Thomas KC, Zheng XF, Garces Suarez F, Raftery JM, Quinlan KG, Yang N. Evidence based selection of commonly used RT-qPCR reference genes for the analysis of mouse skeletal muscle. PLoS One. 2014;9(2):e88653.

27. Reboucas EL, do Nascimento Costa JJ, Passos MJ, de Sousa Passos JR, van den Hurk R, Viana Silva JR. Real Time PCR and Importance of Housekeepings Genes for Normalization and Quantification of mRNA Expression in Different Tissues. Braz Arch Biol Techn. 2013;56(1):143-54.

28. Chapman JR, Waldenstrom J. With Reference to Reference Genes: A Systematic Review of Endogenous Controls in Gene Expression Studies. PLoS One. 2015;10(11):e0141853.

29. Kosir R, Acimovic J, Golicnik M, Perse M, Majdic G, Fink M. Determination of reference genes for circadian studies in different tissues and mouse strains. BMC Mol Biol. 2010;11.

30. Xu L, Ma X, Cui B, Li X, Ning G, Wang S. Selection of reference genes for qRT-PCR in high fat diet-induced hepatic steatosis mice model. Mol Biotechnol. 2011;48(3):255-62.

31. Matouskova P, Bartikova H, Bousova I, Hanusova V, Szotakova B, Skalova L. Reference genes for real-time PCR quantification of messenger RNAs and microRNAs in mouse model of obesity. PLoS One. 2014; 9(1):e86033.

32. Li X, Qiao J, Yang N, Mi H, Chu P, Xia Y. Identification of Suitable Reference Genes for Normalization of Real-Time Quantitative Polymerase Chain Reaction in an Intestinal Graft-Versus-Host Disease Mouse Model. Transplantation Proceedings. 2015;47(6):2017-25.

33. Medrano G, Guan PH, Barlow-Anacker AJ, Gosain A. Comprehensive selection of reference genes for quantitative RT-PCR analysis of murine extramedullary hematopoiesis during development. Plos One. 2017;12(7).

34. Lucas ES, Watkins AJ, Cox AL, Marfy-Smith SJ, Smyth N, Fleming TP. Tissue-specific selection of reference genes is required for expression studies in the mouse model of maternal protein undernutrition. Theriogenology. 2011;76(3):558-69. 